Voix et Images

voixetimages

\title{
Services secrets québécois. Les espions de la paralittérature des années 1940-1960
}

\section{Paul Bleton}

Volume 18, numéro 1 (52), automne 1992

Les écritures masculines

URI : https://id.erudit.org/iderudit/201004ar

DOI : https://doi.org/10.7202/201004ar

Aller au sommaire du numéro

\section{Éditeur(s)}

Université du Québec à Montréal

\section{ISSN}

0318-9201 (imprimé)

1705-933X (numérique)

Découvrir la revue

\section{Citer cet article}

Bleton, P. (1992). Services secrets québécois. Les espions de la paralittérature des années 1940-1960. Voix et Images, 18(1), 118-141.

https://doi.org/10.7202/201004ar
Résumé de l'article

Résumé

On évoque ici un corpus de récits paralittéraires publiés sous forme de fascicules. Production singulière par son thème dominant, l'espionnage, si on se remémore qu'elle fut quasi exclusivement le fait d'auteurs \québécois) singulière aussi par sa stricte circonscription dans le temps (les décennies 1940-1960); singulière enfin par les déterritorialisations esthétique, communicationnelle et thématique dont elle fut le laboratoire. 


\title{
Services secrets québécois. Les espions de la paralittérature des années 1940-1960*
}

\author{
Paul Bleton, Télé-Université
}

\begin{abstract}
On évoque ici un corpus de récits paralittéraires publiés sous forme de fascicules. Production singulière par son thème dominant, l'espionnage, si on se remémore qu'elle fut quasi exclusivement le fait d'auteurs québecois; singulière aussi par sa stricte circonscription dans le temps (les décennies 1940-1960); singulière enfin par les déterritorialisations esthétique, communicationnelle et thématique dont elle fut le laboratoire.
\end{abstract}

\section{Pulps et spionspiel: l'espionnage fasciculaire}

Livres de poche, magazines, fascicules... corpus gigantesquè et encore mal connu de la littérature de grande diffusion publiée dans le Québec des années 1940-1960 d'où, improbable, émerge un héros couleur muraille, l'espion de papier. Trait d'époque sans doute que son succès puisque, dès la fin des années quarante, il s'observait aussi bien aux États-Unis qu'en Angleterre ou en France; trait d'époque donc, qui ne doit toutefois pas cacher l'étonnante originalité du phénomène: son amplitude ${ }^{1}$, son caractère imprévisible, la singularité

- La bibliographie a été constituée par Richard Saint-Germain et Paul Bleton. La recherche dont ce texte est une émanation a été rendue possible par une subvention du CRSH.

1. Entre 1947 et 1966,945 titres pour la seule série IXE-13, près de trente mille pages, pour un tirage hebdomadaire de trente mille exemplaires, ce qui équivaudrait a un total de vingt millions d'exemplaires; n'oublions pas non plus que chaque fascicule avait plusieurs lecteurs, ni le taux de pénétration tout à fait exceptionnel de cet espionnage fasciculaire - $15 \%$ du lectorat - ni la réussite d'IXE-13 - lu par $85 \%$ des lecteurs de fascicules... (Sur ces évaluations quantitatives, $c f$. Vincent Nadeau et Michel René, . Vingt ans de commerce et d'industrie culturelle: jalons pour situer l'importance du tirage des aventures étranges de l'agent IXE-13 -, Eitudes littéraires, vol. XII, n², août 1979). 
de son inspiration dans la littérature québécoise, et sa durée météorique $^{2} \ldots$

Au-delà de la surprise, il est certainement possible de comprendre ce phénomène de façon plus systémique. Tout d'abord, en rappelant que la littérature industrielle québécoise des années 1940-1960 avait eu un précurseur dans les années vingt: le *nationalisme littéraire de masse * de Garand.

Les romans canadiens sont écrits par des Canadiens, imprimés par des Canadiens, avec du papier canadien, illustrés par des Canadiens et édités par des Canadiens pour le bénéfice des Canadiens ${ }^{3}$.

C'est ce que martelait l'encart répété dans les *Romans canadiens" depuis 1923, collection fondée sur le format du fascicule. L'expérience à la fois technico-commerciale et idéológique des * Romans canadiens * de Garand n'allait pas être perdue; le transfert de connaissances vers la nouvelle industrie fasciculaire des années 1940-1960 allait en effet s'effectuer par le truchement d'Alexandre Huot, ex-auteur Garand qui, associé à E. L'Archevêque, devait fonder Le Bavard, devenu ultérieurement Police-Journal, éditeur d'IXE-13...

En outre, largement surdéterminé par l'intention propagandiste, ce premier modèle allait s'accommoder très naturellement d'un second, venu lui d'un autre média de masse, la radio. L'effort de guerre devait aussi porter sur les consciences, et s'incarner dans des feuilletons radiophoniques à partir de $1942^{4}$; feuilletons comme La Flancée du commando qui allaient vulgariser l'univers de la guerre secrète et un nouveau type de héros, le soldat de l'ombre, certes explicitement issu du commando du feuilleton de P. Gury et donc de l'épisodique raid de Dieppe, mais plus durable, plus généralement adapté à la longue durée, au quart de siècle de guerres secrètes à venir.

On le verra, le caractère fasciculaire de l'espion de papier a été largement surdéterminé par la place de l'édition paralittéraire québécoise dans le marché de l'édition francophone; son univers de référence, le spionspiel, était empruntés, comme pour faire antithèse au

2. Multiplication fulgurante des éditeurs et des collections, et disparition tout aussi rapide, une vingtaine d'années plus tard.

3. L'expression est de C.-M. Gagnon, sLes éditions Édouard Garand et la culture populaire québécoise ,, Voix \& Images, vol. X, n' 1, automne 1984..

4. Cf. René legris, Propagande de guerre et Nationalisme dans la radiodiffusion (1939-1955), Montréal, PUQ, 1972; Pierre Pagé et Renée Legris, Répentoire des cenvres de la littêrature radiopbonique québécoise, 1930-1970, Montréal, Fides, 1975.

5. Même si les thèmes abordés ne devaient pas grand chose à l'espionnage français, britannique ou américain de l'époque. 
roman de la terre. Nouveau dans la littérature québécoise ${ }^{6}$, le rôle de l'espion ou du contre-espion n'était pas pour autant inconnu de ses lecteurs des années trente; du moins de ceux qui fréquentaient un peu les pulps et dime novels américains, l'espionnage littéraire ou populaire britannique, éventuellement en traduction 7 , ou les romans français parfois publiés au Québec en vertu d'ententes éditoriales ponctuelles $^{8}$. Une fois implanté par le succès d'IXE-13 qui fit ici office d'œuvre codante, le genre allait se reproduire presque de lui-même, jusqu'à ce que les conditions de marché qui avaient permis son apparition soient modifiées, dans les années soixante. C'est ce quart de siècle qui fut l'âge d'or du roman d'espionnage dans la paralittérature québécoise; avant les fascicules, rien; après eux, pas grand-chose. Certes, on devait écrire des romans d'espionnage plus tard... une vingtaine peut-être, si l'on fait un rapide survol et que l'on se donne une définition libérale: on voit que l'espion de papier s'est étroitement identifié à un moment de la littérature québécoise - épisode très circonscrit dans l'histoire de la production culturelle?

Étroitement identifié à ce moment, circonscrit, de la littérature québécoise, disions-nous, mais aussi au seul support du fascicule. Bien sûr les fascicules ont servi de véhicule à d'autres genres, le sentimental, le policier, le western... Mais, au moins dans les fascicules cherchant un lectorat masculin, en dépit de l'étroitesse de son univers de référence, l'espionnage a été l'une des inspirations les plus fécondes de la période.

En outre, déséquilibre notable, l'espionnage n'investissait pas le format-livre, alors qu'il était sur-représenté dans les fascicules ${ }^{10}$. Les

6. Il y eut toutefois quielques précurseurs thématiques comme La Valise mystérieuse (Montréal, Edouard Garand, coll. - Roman canadien inédit, 1930), de J.-M. Lebel, ou Les Amours de William Benjamin (Montréal, Edouard Garand, coll. - Roman canadien inédit, 1931) de Jean Feron.

7. Le prix Nobel Kipling, les best-sellers Oppenheim ou Wallace, le gouverneur général Buchan, le grand-écrivain Maugham et l'ouvre codante d'Ambler...

8. Je pensais à la série Ceux du SR, de Ch. Robert-Dumas, auteur de Fayard imprimé a Montréal par les éditions Variétés de Péladeau; mais cette édition québécoise date en fait de 1946.

9. On se contentera de mentionner les curieux arrangements commerciaux qui font que de l'espionnage occasionnel apparaît dans certains volumes sans date de la collection -Le jaguar rouge, avec des auteurs français (comme $H$. Ghilen), un imprimeur italien et un éditeur montréalais (Presses sélect)!

10. À l'exception de Nora l'énigmatique (Montréal, Pascal, 1945) signé Pierre Hartex, pseudonyme de P. Daignault, de quelques traductions de l'anglais dans la collection -Petit-format. et de quelques romans-jeunesse comme Les Mystères du croiseur Toulouse (Montréal et Paris, Fides, 1957) de G. Cerlebaud-Salagnac ou Max (Québec, Éditions jeunesse, coll. -Jeunesse, 1965) de Monique Corriveau. 
importations, très minoritaires dans les fascicules et très majoritaires dans les livres, n'y illustraient l'espionnage que de façon très sporadique ${ }^{11}$... Enfin, dans cet âge d'or des magazines, certains, notamment Mon magazine policier et d'aventures, servirent de vecteur, mais de vecteur occasionnel seulement, à des histoires d'espionnage parfois commandées à des auteurs québécois, le plus généralement importées...

En résumé, l'espionnage évoqué ici a été un phénomène essentiellement fasciculaire, québécois et circonscrit à la période 1940-1960.

Rupture dans l'approvisionnement du fait de la guerre (et de l'immédiat après-guerre), ingéniosité d'imprimeurs qüi s'improvisaient éditeurs pour emplir leurs bons de commande, *capacités de production accrues depuis la crise et "possibilités d'approvisionnement en papier de type neus, éphémère et peu coûteux ${ }^{12}$ : tous ces facteurs ont contribué à l'émergence de cette paralittérature. Et les livres de poche importés en particulier les Marabout - lui ont porté un coup dont elle ne s'est pas remise dans les années soixante. Mais l'âge d'or de l'espionnage québécois aura duré deux décennies, malgré la relation de pouvoir économique inégale entre le centre et la périphérie francophonique, parce qu'il sut justement utiliser le format-fascicule que la littérature populaire française d'après-guerre devait très 'rapidement désinvestir, laissant du coup un créneau, permettant une complémentarité des formats.

Nouveau format universel dans la paralittérature du temps, le livre de poche, inventé en 1935 par Penguin puis en 1939 par Simon \& Schuster, acclimaté à partir de 1953 par Hachette en France, le livre de poche donc se présentait sous les apparences d'une miniaturisation du format, d'un coloriage de la tranche, de l'exhibition du contenu par une illustration colorée chargée de provoquer l'acheteur potentiel, sans que le contenu change; cette démocratisation des Belles-Lettres, c'est l'acceptation par le support que la Littérature est - aussi - une affaire économique. Mais à côté des Belles-Lettres démocratisées, réemballées, ce nouveau format, renforcé par quelques simplifications techniques, allait permettre aux collections populaires de radicalement changer leur plumage: couverture non pelliculée dans les premières

11. Traductions comme Sinistres Rendez-wous (Montréal, Éditions de la revue moderne, 1949) de P. Cheyney, comme Je ne suis pas un béros (Montréal, éditions Moderne, coll. . Petit-format., 1951) d'E. Ambler ou de quelques romans de Van Wyck Mason, série fasciculaire à l'espionnage occasionnel comme - Les aventures de Bill Disley. de J. A. Flanigham, série romanesque comme * MissTéria - de M. Allain.

12. Cf. Louise Milot, Vincent Nadeau et Denis Saint-Jacques, . Du plaisir à 10 cents -, Cabiers pour la littérature populaire, n*0 8-9, 1987. 
années, illustration à deux couleurs, tranche non jaspée, tirage sur rotative et non plus à plat, brochage automatique ${ }^{13}$...

La paralittérature québécoise 1940-1960 avait fait usage de ce format poche très tôt, presque dix ans avant la France - la première collection, *Petit-Format", vécut de 1944 à $1958^{14}$. Le modèle était bien entendu le paperback' américain, premier avatar, aujourd'hui disparu ${ }^{15}$. Au moment où devait se faire sentir le déclin de l'empirepulps $^{16}$, les auteurs auraient éventuellement pu s'adapter au format livre de poche; ce qui ne se produisit pas: reconversion manquée? inspiration trop courte d'écrivains trop conditionnés par le 32 pages canonique? cultures et intérêts incompatibles des éditeurs de livres et de ceux de fascicules ${ }^{17}$, entre des importateurs, des pirates parfois et des instigateurs de. création originale et autochtone? Toujours est-il qu'alors que le marché changeait, que disparaissait le format fasciculaire en France; en Angleterre ou aux États-Unis, que s'améliorait la qualité du livre de poche populaire, que s'opérait une forte concentration de la production - collections hégémoniques, disparition du pullulement de petits éditeurs qui tentèrent leur chance immédiatement après la guerre - la paralittérature québécoise se cantonnait à répéter les recettes qu'elle avait éprouvées: peu de remaniements de présentation ou d'inspiration pour ses fascicules en vingt ans, peu d'améliorations de présentation ou d'efforts de créations originalès pour ses livres de poche. La comparaison entre les premiers fascicules et les derniers, entre les premiers titres de "Petit-format " et les derniers, révèle cette vision à court terme de l'industrie paralittéraire, sa faiblesse technologique dans le développement du produit.

13. À ce sujet, cf. Frédéric Ditis, -La production du livre de poche en Prance", Bibliograpbie de France. Biblio, $n^{\circ}$ 22, 1973; - Lacclimatation du livre de poche en France., Bibliographie de France. Biblio, $n^{\circ} 24,1974$. Il etait alors directeur de J'ai lu.

14. Sur cette collection, of. Richard Saint-Germain, - La collection Petit Format des Éditions de La Reurue moderne de Montréal ', Encrage, $n^{\circ}$ 18, mars-avril 1988.

15. Sur ce format du premier paperback paralittéraire, on consultera G. O'Brien, Hard-Boiled USA - Histoire du roman noir américain, Amiens, Encrage, coll. - Travaux :, $n^{\circ} 2,1989$.

16. La datation des fascicules ne pouvant être le plus souvent qu'approximative, il est difficile de préciser les débuts de cet espionnage fasciculaire; néanmoins, Espionnage et Contrebande de J. Desgranges, fascicule de 14 pages sans illustration de couverture était publié par les Éditions de l'Étoile en 1942. Une première?

17. Cette hypothèse d'un conflit de culture organisationnelle et d'intérêt commercial entre éditeur de livres et éditeur de fascicule est spectaculairement mis en scène lorsque, comme Police-Journal, on passe de liun a l'autre (en devenant Sogides): l'homme d'affaires qui a réussi veut couper avec la culture à $10 \leftarrow$ qui avait permis l'accumulation initiale de capital. 


\section{Fascicules et figuration narrative}

La multiplication des titres commandée par le support-fascicule devait produire deux effets: tout d'abord, emphatiquement souligner l'effet-série, le caractère sériel de cet espionnage fasciculaire; puis, à cause de la multiplication parallèle des illustrations de couverture, amener cette littérature dans la mouvance d'autres hybridations sémiotiques verbo-iconiques, comme l'affiche, l'image publicitaire ou, surtout, la BD. Qu'une logique évidemment commerciale ait présidé à cette hybridation ${ }^{18}$ n'empêche, en effet, nullement d'inscrire l'illustration fasciculaire dans une histoire des croisements des récits paralittéraires et de l'image - la $\mathrm{BD}$ américaine n'est-elle pas née de la rencontre de la figuration narrative et de la presse Hearst?

Sérialisation de l'apparence, donc; normalisation de chaque couverture. L'occupation de l'espace paratextuel est fortement commandée par des exigences commerciales et cognitives: le renvoi de chaque fascicule à son appartenance sérielle, à son appartenance générique, à son statut de marchandise standardisée. Sur la couverture, environ $40 \%$ de cet espace est dévolu à l'identification de la série par le graphisme généralement le visage du héros sert de logo - et l'intitulation, particulièrement prolixe - comme "les aventures fantastiques de l'agent ZED 29 l'as des as canadiens * ou *le génial espion X-14 agent secret canadien. . Le prix et l'éventuelle numérotation sérielle apparaissent aussi dans ce premier espace. L'illustration et le titre particulier de chaque fascicule occupent le $60 \%$ restant; l'illustration, souvent un lavis; généralement à deux couleurs obtenues par une séparation mécanique (parfois approximative d'ailleurs), sur un papier gloss - un peu plus résistant, permettant une impression plus nette, offrant une apparence plus chic, un dessin - l'illustration donc est parfois signée (L'Archevèque, P. Hardy, Lili...), mais très fréquemment anonyme...

L'illustration de couverture, dans l'esprit des dime novels américains, mais tout aussi bien de la tradition de la littérature populaire depuis le début du siècle, extrait une situation du récit, souvent paroxystique - la "scène à faire*. Intensité pure, provocation à

18. Explicitement, le paratexte fasciculaire sert de véhicule publicitaire; considérez par exemple les quatrièmes de couverture de L'Enfer et de Le Senpent qui annoncent une autre série (celle d'. Arsène Lupien.), celle de Le Nazi une autre série encore (-Rapace .); alors que les dernières pages intérieures annoncent les soldes des Editions Irène (Un nazi maudit et Le Serpent), et que, plus rarement, lorsque le 32 pages canonique était-encore trop vaste pour l'inpiration de l'auteur, un résumé de l'épisode garnissait une page vide (L'Homme-femme, $n^{\circ} 15$ de -L'espion X-14 )... 
l'endroit de l'acheteur prospectif , mais aussi placement du topic: cette illustration est facile à comprendre, à la fois représentative de l'univers de référence du spionspiel, vitrine sur le contenu du fascicule.

Intensité pure dont le récit donnera l'expansion narrative redondante, comme la scène de séduction dans le goût kitsch des publicités de voitures de l'époque (grosse calandre/seins agressifs) à la fois explicite et détournée - caché dans l'arrière-plan et les buissons, derrière le dévoilement de la séductrice et la fascination de sa dupe boutonnée et casquettée, un tiers malveillant menace d'une manière autrement dangereuse le mâle leurré par les Jambes de femme promises par le titre et le dessin (G. Durant Jambes de femmes, coll. * La belle Françoise", n 140 [voir page 125D.

Ou intensité pure elle-même rhétorisée, comme ce commentaire graphique fondé sur la métaphore visuelle du cobra, sortant de la tête d'un homme terrorisé pour lui infliger quelque mortelle et prévisible morsure (A. Prévert, L'Espion aux cent visages, coll. *Madame X- [voir page 126]).

Devant la masse de ces images, deux attitudes possibles: la sensibilité à la répétition ou la sensibilité à l'enchainement, le repérage de thèmes ou le repérage d'une syntagmatique de cette figuration narrative. Laissant à une étude à venir l'analyse de la place de l'illustration fasciculaire dans l'histoire de la figuration narrative, concentrons-nous, à l'aide de trois exemples, sur un des thèmes majeurs de ces illustrations: le regard.

Voir et savoir, ce sont les deux dimensions auxquelles renvoient non seulement l'illustration de couverture en général, mais aussi, exemplairement, sui-référentiellement; ces trois illustrations. Dans l'illustration de Jambes de femme, le lecteur est à la fois voyeur et bon entendeur, ayant à la fois sous les yeux le corps exhibé séduisant l'officier, mais aussi ce que l'officier ne voit pas, l'épée de Damoclès de l'homme armé caché dans un buisson.

Dans L'Enfer de Séoul, le regard se thématise, mais ce regard ne s'adresse pas au lecteur, redoublant par la vignette et l'illustration sa direction fuyante. Dans la vignette, qui se répète donc dans tous les numéros, le héros regarde par dessus son épaule, il est dans un monde dangereux où la méfiance est une vertu; sur l'illustration, il (le même héros sans doute) voit ce que voit le lecteur, une usine d'où s'échappe une épaisse fumée (destructrice). Mais en plus de ce regard qui le définit - regard dérobé en ce double sens qu'il ne nous regarde pas et qu'il regarde l'usine fumer en se planquant, comme un bon espion -, le héros, contrairement au lecteur, est plongé dans l'action, littéralement, puisqu'il nage accroché à un tronc flottant, 

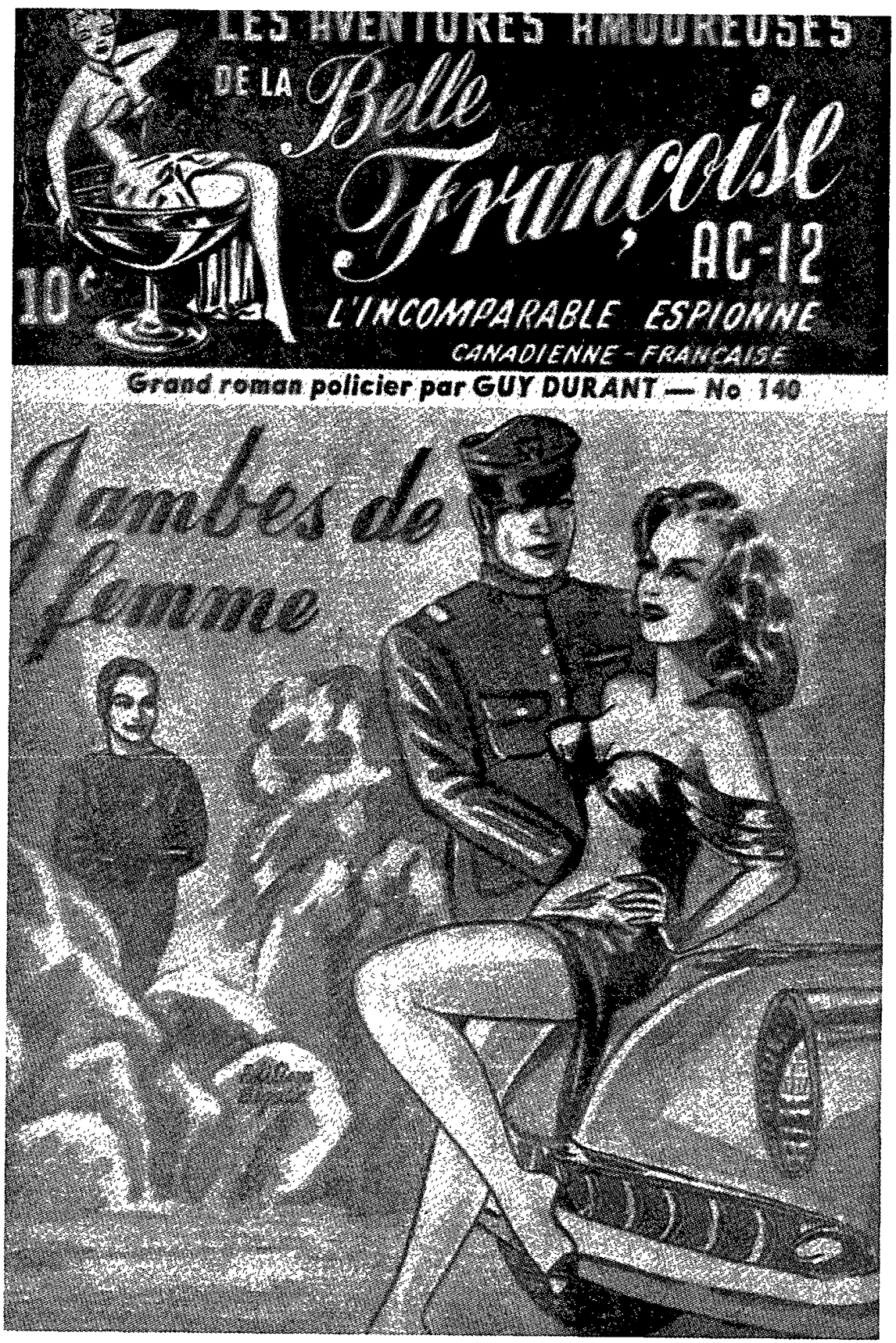

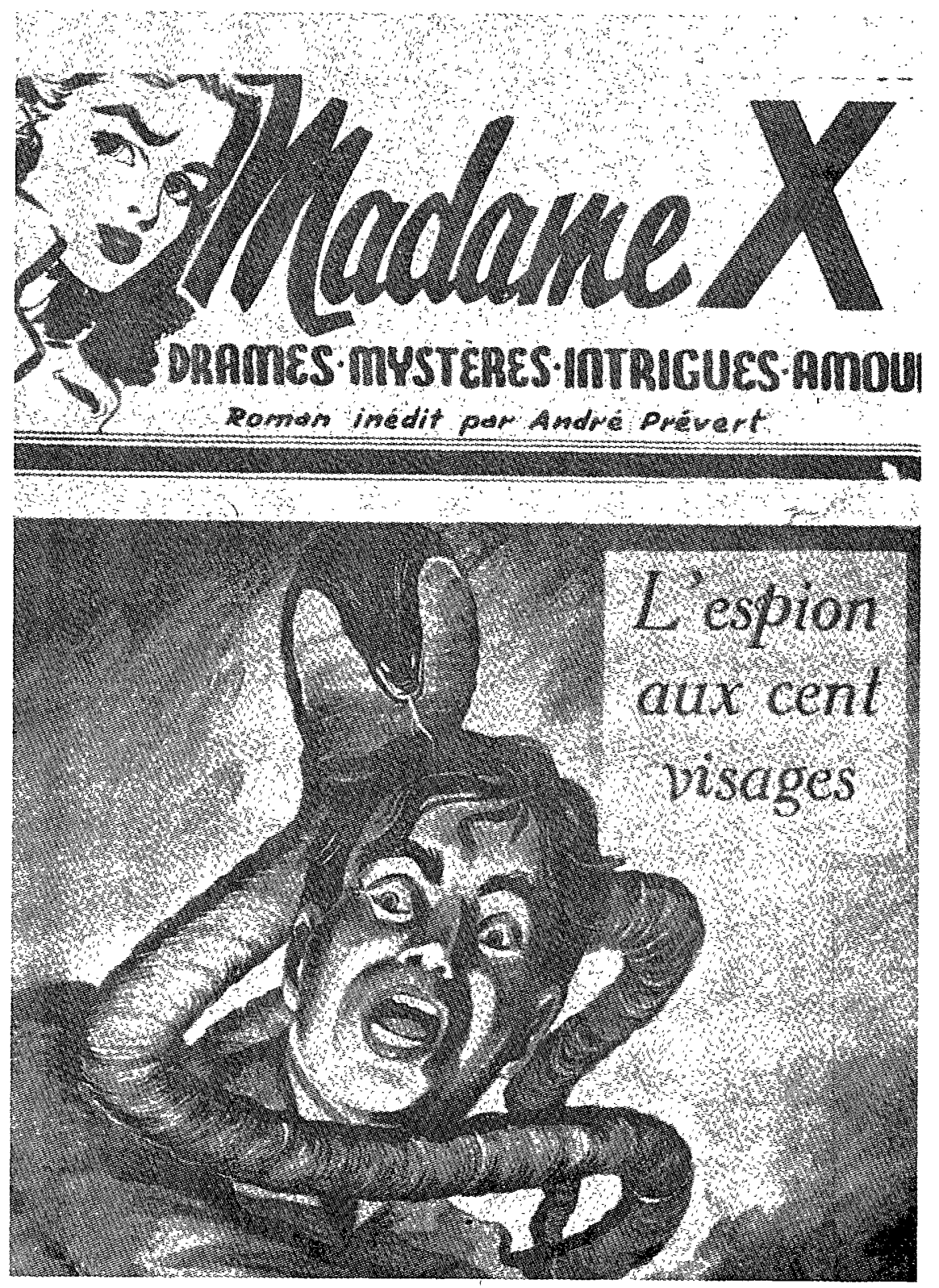
caché sans doute derrière ce tronc, alors que le lecteur nage encore quant aux aboutissants de l'histoire à venir, à défaut de nager sur les tenants, puisque, lecteur sériel, il sait, il connaît ce qui s'était passé avant, il attend dans ce numéro ce que les précédents lui avaient déjà offert, de l'espionnage en Corée. Le récit viendra d'ailleurs confirmer bien des supputations préliminaires, resituant l'image dans le fil d'une histoire de sabotage derrière les lignes adverses. (M. Leroux, L'Enfer de Séoul, coll. "L'agent ZED 29", $\mathrm{n}^{\circ} 18$ [voir page 128]).

Dans Un nazi maudit, le regard de la vignette n'est plus fuyant mais absent. Le bon entendeur ( génial " dit le titre de la série) se mue en entendeur; la vignette thématise plutôt la parole. Regard absent redoublé dans l'illustration par le regard vide (lunettes du masque à gaz) qui évite le lecteur. Regard de qui, d'ailleurs? Ami? ennemi? X-14 ou nazi maudit? Regard et identité occultés. En outre, la pure intensité produit ici une stéréosémie, un sens obvie et des sens obtus. Le sens obvie - le danger et la violence, le pistolet, le tuyau crevé; et sens obtus: à qui sont les poings du premier plan? ceux d'un homme couché? poings de combat ou crispation de douleur? quelles sont ces étincelles? des feux d'artifice externes? phosphènes, perçus justement quand la paupière est fermée (Anonyme, Un nazi maudit, coll. "L'espion X-14*, n 9 [voir page 129])?

Dans Le Serpent de Formose, la couverture n'a plus de vignette, plus de sceau sériel insistant sur le regard donc; par contre, l'illustration ellemême cette fois met le lecteur face à trois regards frontaux, imbriqués, formant un mur, impénétrable(s) - bien sûr puisque asiatique(s), le point de fuite lui-même ne permettant pas la fuite, le détournement du regard: la pagode d'arrière-plan ramène le lecteur à la sinité. Et comme l'impénétrabilité de cet exotisme pourrait laisser le lecteur perplexe sur la valeur à y accorder (les lèvres de la belle chinoise sont bien attirantes, bien invitantes), le titre se charge de remettre en circulation la maxime pragmatique bien connue du lecteur de romans d'espionnage, déjà activée dans cette autre illustration dans laquelle la séduction féminine fournissait le topic - Jambes de femme - "les apparences sont trompeuses". Par rapport à ce que le lecteur trouvera à l'intérieur, il ne s'agit pas dans cette image d'une scène du récit à venir, pas non plus d'une métaphore comme celle de L'Espion aux cent) visages, mais d'une perception globale de l'altérité asiatique, fascinationn et danger, directement adressés au lecteur et non plus à un personnage (Anonyme, $L e$ Serpent de Formose, coll. "L'Espion n ${ }^{\circ} 13$ ", $\mathrm{n}^{\circ} 10$ [voir page 130]).

Comme on le voit, l'image de couverture accroche, incite à la lecture, donne de l'information préalable, titille la curiosité en ouvrant 


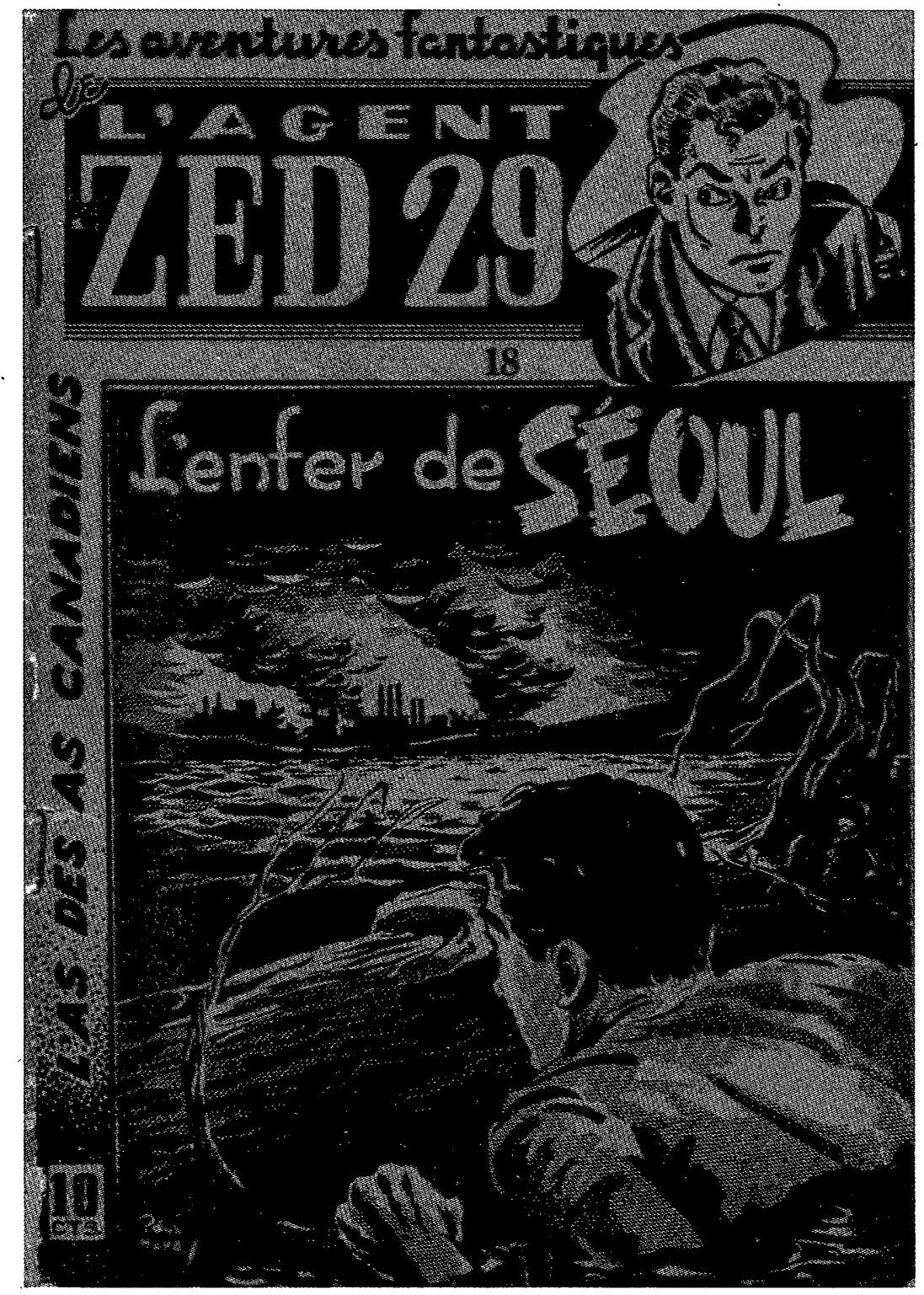




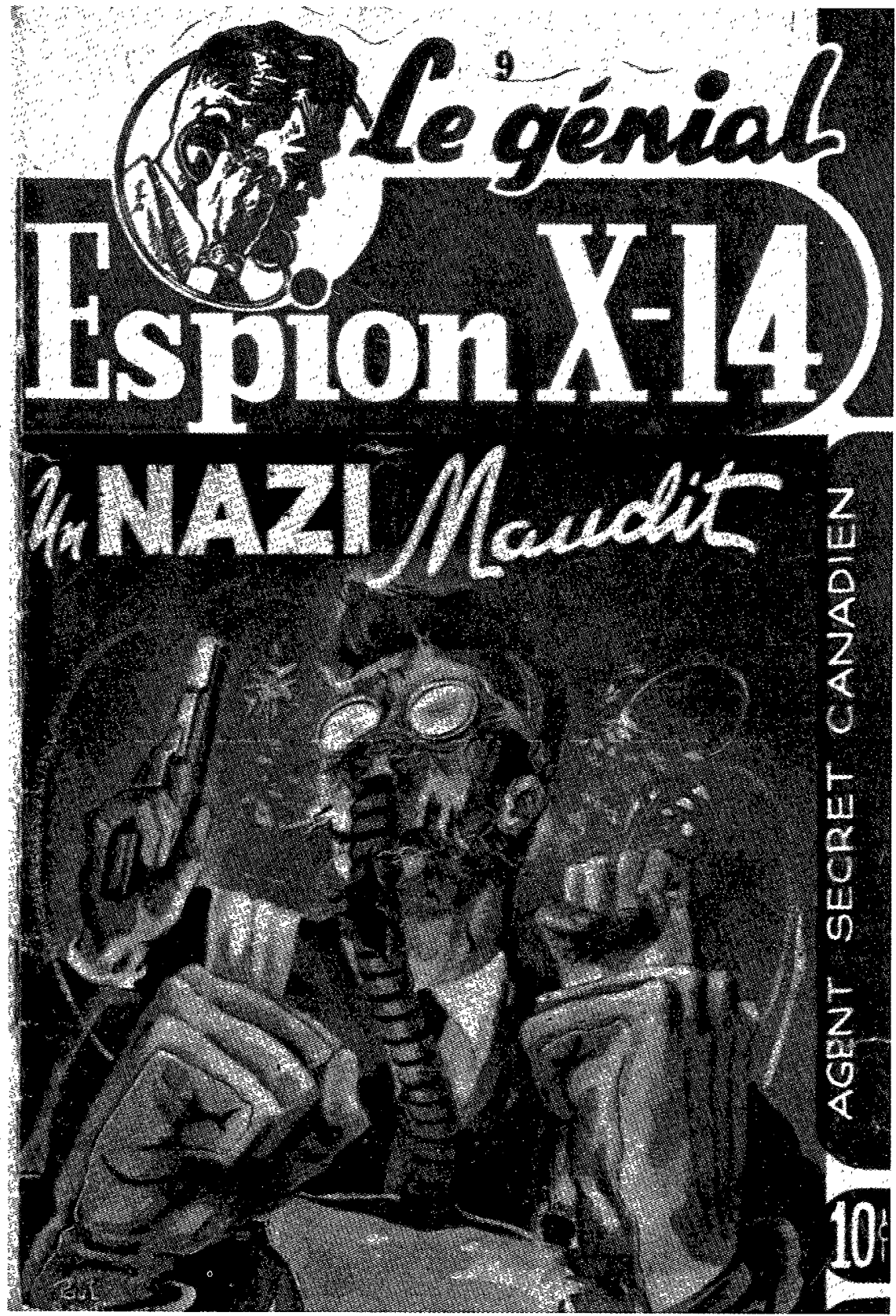




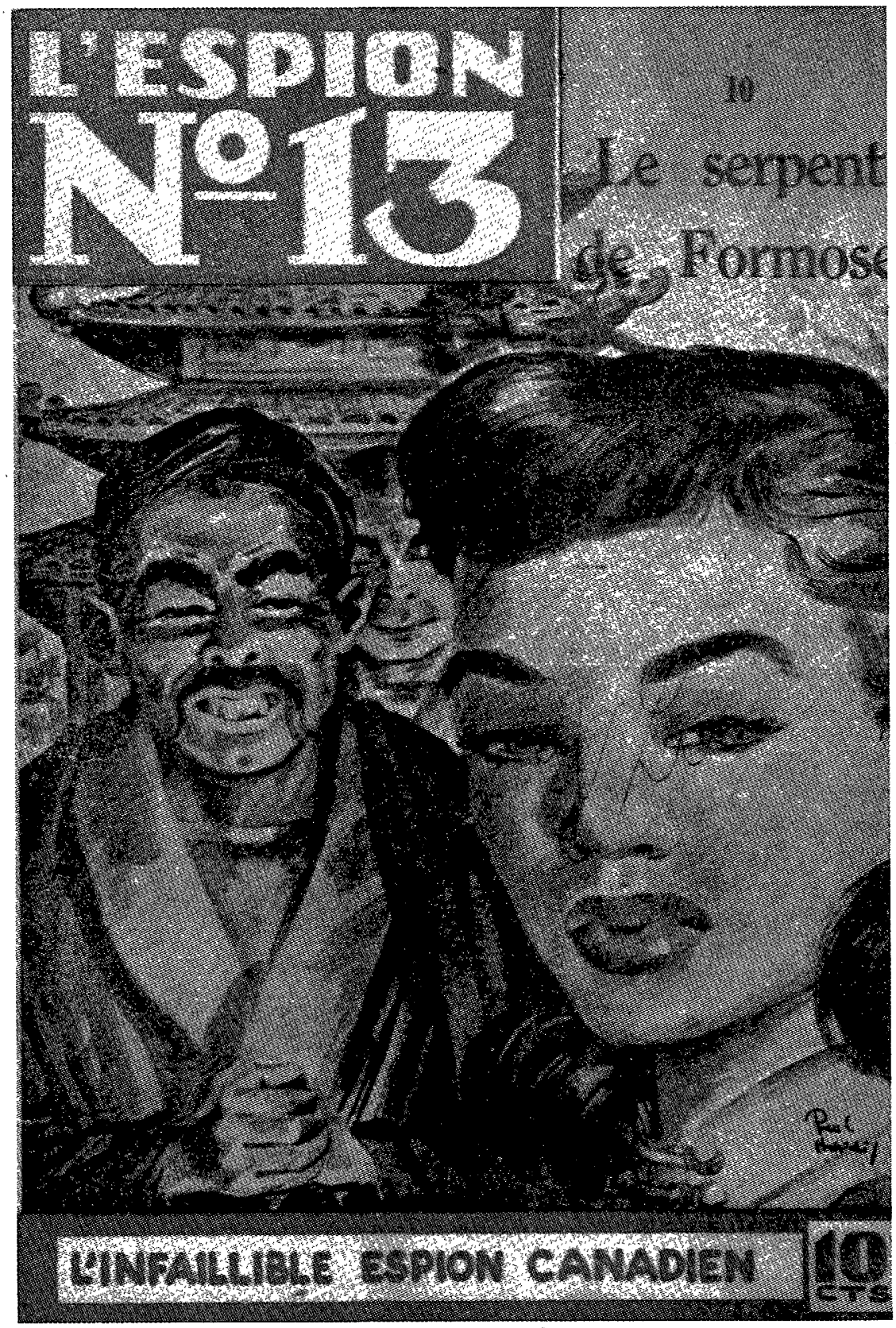


quelque "code herméneutique", fait rêver peut-être: elle est partie intégrante de l'acte de lecture.

\section{Stratégies de facilitation de lecture}

L'effet-série était renforcé du fait que le succès d'une série appelait d'inévitables créations épigonales, dans les titres de série, dans les apparences paratextuelles, dans l'inspiration; ressemblance bien sûr explicitement recherchée, ou *comment rendre certains de ces produits interchangeables dans l'esprit de l'acheteur *?

Mais outre leur fonction commerciale, la ressemblance entre séries et la répétition dans une série ont une incidence cognitive majeure: la forte prévisibilité de l'histoire que l'on s'apprête à lire, donc sa plus grande facilité. Nul doute, le succès de cette paralittérature a été fonction de ses stratégies de facilitation de lecture.

"L'écriture secrète", "la formule volée"; "la lentille qui tue", "mission de mort "; "mystification ", .l'espion aux cent visages"; * elle avait trop d'amants", "harem de beautés"; .le nègre de Bali", .le serpent de Formose .... la fourchette lexicale des titres ne risque pas de prendre le lecteur au dépourvu; tel est bien l'univers de référence du genre: secret, violence et action, tromperie, érotisme, exotisme... Aussi bien, précoce annonce du sujet (topic) et forte redondance sontelles des stratégies élémentaires de facilitation de lecture.

Dans une série comme :l'Agent k-7, célèbre espion international , le héros est aussi narrateur intradiégétique, à qui le narrateur du récitcadre passe la parole - le narrataire du récit-cadre est le lecteur; le narrateur de ce récit-cadre et son épouse devenant du coup les narrataires intradiégétiques du récit encadré. Or, de telles (relatives) complexités narratologiques ne sont pas coutumières des autres séries. Ainsi, le style indirect libre, zone traditionnelle de jeu entre les niveaux de l'énonciation, intervient relativement peu, et sous haute surveillance; pas question par exemple qu'il serve à brouiller la source de l'énonciation ${ }^{19}$. Le recours répété à une coopération interprétative explicite n'est pas non plus très fréquent, et lorsque le cas se présente, dans les passages :salaces* de La Fin d'un espion, de Lydia Mitchell

19. Un exemple: :L'espion $n^{\circ} 13$ questionnait adroitement la danseuse. Quels étaient les amis de Bert?... Quels lieux frequentaient-ils à part ses affaires et son commerce?... Est-ce qu'il avait des ennemis?... Est-ce qu'on pouvait raisonnablement soupçonner quelqu'un? Qui avait-il fréquenté parmi les gens suspects de terrorisme? Quel rouge pouvait-on soupçonner?... Le Serpent de Formose, p. 19. 
par exemple, la contribution demandée au lecteur reste modeste ${ }^{20}$. La narration à la première personne n'est elle-même pas très fréquente ${ }^{21}$.

La lecture d'un fascicule comme Tina-Gogo ${ }^{22}$ est représentative de ce que le lecteur devait fournir comme effort cognitif. Dans les deux premiers motifs, l'embuscade et l'énonciation du mandat de Françoise, le point de vue est instable: c'est visiblement davantage le souci de donner le plus rapidement possible de l'information au lecteur que celui de la cohérence du point de vie qui comptait dans l'enchaînement à un énoncé comme "Un jeune homme accroupit [sic] dans l'herbe d'un champ, examine l'horizon d'une paire de jumelles"; un autre comme * On dirait qu'il est sur le point d'accomplir quelque chose qu'il a attendu durant de longs mois *. D'autant que la narration veut cacher pendant quelques pages encore l'identité de l'homme en embuscade - un fiancé un peu délaissé par AC-12, et depuis circonvenu par une belle espionne ennemie. Lorsque le récit doit rendre une situation intense ou un vif enchaînement d'actions, l'accélération rythmique comme choix stylistique se laisse largement surdéterminer par la propension des auteurs à tirer à la ligne. Qu'on me permette de citer in extenso la page 13, dans laquelle Françoise est de nouveau la cible du tireur inconnu:

Bang!... Bang!...

Deux coups de feu.

Les balles lui frisent les cheveux.

La jeune femme se met à courir.

Elle descend une pente.

L'eau de la rivière est juste devant elle.

- Bang!

Une troisième balle touche le bras de l'espionne.

Son sang coule.

La blessure n'est que superficielle.

Le projectile n'a qu'éffleuré la peau dorée d'AC- 12 .

C'est pourtant assez pour lui faire faire le plongeon.

Elle saute à l'eau.

La rivière n'est pas profonde.

Mais la belle Françoise est emportée par le courant.

Le jeune homme au revolver arrive sur la rive.

20. Lydia Mitchell, La Fin d'un espion, Montréal, P.B., coll. *Les aventures de Ray le justicier , [s.d.]. Avec des points de suspension servant a marquer une réticence bienséante (.Paulette était assez menue mais sés... rondeurs étaient des plus... tentantes... - p. 11 ; - mais qui nous empêche de penser aussi à... ce que nous ferons... quand nous aurons terminé [notre travail] ;, p. 20).

21. Comme dans : La mission secrète - de J. Berville.

22. M. Lancret, Tina-Gogo, Montréal, Éditions Bigale, coll. -Aventures amoureuses de la belle Françoise, AC-12, $n^{\circ} 307$, [s.d.]. 
Il voit sa victime lui échapper.

Il vise.

- Bang!

La balle fait plonger AC-12.

Elle nage sous la surface.

Mais elle ne va pas assez vite.

Ses vêtements l'encombrent.

Elle oublie tout de sa modestie.

D'un geste vif, elle enlève ses vêtements.

Elle en fait un colis qu'elle passe rapidement autour de son poignet.

Elle peut nager plus rapidement.

Le jeune homme au revolver voit tout ça.

Il voit encore mieux.

L'homme est plongé à l'eau [sic].

Le revolver est au fond de la rivière.

- Tu ne m'échapperas pas comme ça AC-12!

Il a la haine écrite dans le visage.

Il veut la tuer de ses propres mains.

L'autre jeune homme, son frère, arrive à son tour sur les lieux.

Les coups de feu lui ont fait peur.

Il ne veut pas que son frère ait tué Françoise.

On le constate, la page ne succombe pas sous le poids d'une surabondance verbale, et le phrasé s'en tient aux recommandations de rédaction de l'école primaire; plutôt que la complexité introduite par la subordination syntaxique (relative, complétive...), on préfère ici la parataxe, la "décompacification * (la phrase en "mais" est séparée de celle la précédant); la redondance permet, elle, de ne jamais se tromper sur l'interprétation à donner à l'onomatopéïque * bang *!...

L'auteur ne se prive pas d'effets de surprise; mais l'éventuelle opacité sémantique qu'ils introduisent pour le lecteur doit rapidement être levée ${ }^{23}$.

Ces stratégies de facilitation de lecture ont pu être décrites comme de simples défaillances d'écriture. Il est vrai que pour un lecteur au capital culturel plus fourni que celui du lecteur sériel, les défauts généraux de toute paralittérature - comme tirer à la ligne - les évidentes maladresses d'une industrie débutante aggravées par les conditions de production - l'indigence de l'illustration souvent, l'évidente absence

23. Ainsi, Tina Gogo en vient à pointer son revolver sur le front d'AC-12; celle-ci même manque d'optimisme sur la suite des événements (-Françoise fait ses prières. Elle admet que sa vie est sur le point de prendre fin [...] $)$; et le pire semble bien advenir (-Bang!... Le corps de la jeune femme se plie en deux. La balle l'a atteinte en plein front. .)... Que le lecteur se rassure, il ne faudra que quelques lignes pour découvrir que - le corps de la jeune femme. était celui de la scélérate Tina Gogo! 
d'édition, la connaissance approximative du code orthographique... tout ceci incite à la moquerie ou à la sévérité ${ }^{24}$. Toutefois, une telle réduction des fascicules à des fautes qui visiblement ne décourageaient en rien le lectorat visé serait le meilleur moyen de méconnaître le statut du linguistique dans cette production paralittéraire.

\section{La langue des fascicules}

Selon l'hypothèse tétraglossique d'Henri Godard ${ }^{25}$, le Québec sociolinguistique de cette période pourrait se décrire comme la coexistence parfois conflictuelle de vernaculaires divers, d'une lingua di pane à Montréal surtout, l'anglais, créolisant le français de l'industrie et des techniques, d'une langue prestigieuse, le français des collèges classiques et d'une langue pour le contact avec l'au-delà, le latin. Or, l'effort linguistique de la paralittérature fasciculaire portait sur la promotion et la complexification d'un seul de ces niveaux glossiques, le véhiculaire, pas anglais toutefois, ni joual, langue des fascicules plutôt, création esthétique originale. Avec pour effet la dévaluation de facto des trois autres niveaux glossiques, le vernaculaire, le français des collèges classiques et lè latin, redistribués sous forme de traces dans le véhiculaire fasciculaire.

Entre le vernaculaire du village d'origine et le français de collège classique de la bourgeoisie urbaine, l'espionnage fasciculaire et son lectorat se retrouvaient dans une langue à la fois dynamique parce que minimaliste et incertaine parce qu'hybride. Assurance de cette langue sans éditeurs, sûre de son adaptation à dire l'action la plus échevelée, consciente de son succès; malaise de cette langue écrite, que les contraintes linguistiques de la narration historique insécurisaient - passés simples et concordances de temps. De plus, ces insécurités stylistiques étaient redoublées par la tentative de créer un vernaculaire urbain à partir d'importations paralittéraires. Insécurité face

24. Un petit florilège, pour faire toucher du doigt ce dont il est question: attribution fautive d'une illustration, ou recyclage d'une illustration déja utilisée ailleurs, comme dans Mission de mort, l'beure zéro, Berthierville, Editions Irène, coll. sLes aventures extraordinaires de l'espion $n^{\circ} 13$, l'infaillible espion canadien , $n^{\circ} 204$, [s.d.]; erreur sur le titre en pleine couverture: ede mort mission. au lieu de -mission de mort '; coquilles et curiosités orthographiques, grammaticales et autres: : la balle était destinée pour vous :, : Rosine souria également, , voyons, voyons chérie, un peu de décense., - je pensais de la connaître., "On a eu la preuve que ses transactions avec l'ennemi fut la cause de la mort de militaires et de soldats. [ce qui aggrave sans doute son cas], -alors qu'il leva les bras [...]-, euphémisme par inadvertance: : j'offris donc ma décision. [pour - ma démission.]...

25. Henri Godard, L'Aliénation linguistique (analyse tétraglossique), Paris, Flammarion, 1976. 
au français de collège classique qui sous forme de stylèmes mal intégrés faisait retour dans le style minimaliste.

Traces du niveau glossique prestigieux lorsque l'on manie l'écriture. Traces dont l'envers serait, à cause des dialogues, un style parlé tout aussi indécis, approximation du style dur-à-cuire des pulps policiers américains, mâtinée d'argotismes série noire, bien éloignée du joual urbain de l'époque. Parfois de curieuses instabilités du registre du dialogue en résultaient, pas toujours commandées par une intention parodique.

Ces usages de la langue s'accompagnaient d'une théorie spontanée du langage reconstituée à partir des affleurements du niveau métalinguistique ici et là. Ainsi, l'insistance sur la forme, le mot "mot * dans la phrase .le mot "mort" se passait de bouche en bouche [...]", est en parfaite contradiction avec un silence du texte: le lecteur ne sait pas en quelle langue ce mot est proféré - russe, ukrainien? - ni même s'il est compris par l'espionne parachutée en Ukraine ${ }^{26}$. Au diable la vraisemblance d'ailleurs; c'est la neutralisation, l'indécidabilité de la réponse à cette question qui fait ici office de symptôme; ceci nous dit en effet que dans cette théorie spontanée, la langue n'est rien d'autre qu'un outil de communication, pas une forme signifiante. Même lorsque l'opacité signifiante est en cause, qu'elle se thématise explicitement, la compréhension du lecteur n'est en rien affectée:

Elle entra dans la cabine et eut le temps de demander Sam:

- 'Vite, viens me rencontrer à la porte de sortie et armé, j’ai quelque chose d'intéressant.

Olga naturellement avait parlé en code et si quelqu'un avait écouté la conversation ça n'avait rien de compromettant ni d'intéressant ${ }^{27}$.

Parfois quand même, au nom de la vraisemblance sans doute, l'univers' de référence, international par définition, contraint les héros au polyglottisme. Polyglottisme de pure convention, certes, mais toujours hyperbolique ${ }^{28}$.

26. G. Durant, Jambes de femme, Montréal, Éditions Bigalle, coll. - Aventures amoureuses de la belle Prançoise., $n^{\circ} 140$, [s.d.], p. 26.

27. Anonyme, L'Homme-femme, Berthierville, Editions Irène, coll. -L'espion X-14", $n^{\circ} 15$, [s.d.].

28. - De sa cachette il entendait les hommes parler entre eux et pouvait facilement saisir leur conversation. Parfois on parlait en anglais, le plus souvent en allemand et le génial espion avait même saisi des phrases en italien. Ca le faisait rire, lui qui parlait couramment plusieurs langues, entre autre, le français et l'anglais, le polonais et le russe, plusieurs dialectes tchèques, l'allemand, l'italien, l'espagnol et combien d'autres langues et axiomes [sid]. - (Un nazi maudit, p. 4) 
Hyperbole narrativisant une survalorisation du polyglottisme et correspondant à l'absence de l'ineffable mystique ou du sacré dans cette théorie spontanée, conception communicologique du langage correspondant à l'effacement de la contradiction politico-linguistique entre les deux langues officielles: on est effectivement loin de ce que le collège classique pouvait instiller comme théorie du langage à la même époque dans l'esprit de la jeune bourgeoisie.

\section{Une lecture à géométrie variable}

Stratégies de facilitation de lecture, avec l'instauration d'une langue minimaliste; instabilité sociolectale dûe à la fois à la survalorisation du niveau glossique véhiculaire, pourvu d'une théorie du langage spontanée, et à l'annexion par le véhiculaire de traces du français des collèges classiques et d'un vernaculaire intertextuel: à ces observations poïétiques, valides pour l'émetteur, adjoignons quelques remarques esthésiques, centrées sur le récepteur, et en particulier voyons les incidences du format sur la lecture.

La matérialité même du 32 pages canonique permet en effet de préciser le portrait conjoint de cette paralittérature et de son lecteur. Les auteurs n'ont évidemment pas inventé le format, c'est une contrainte du pliage pour l'imprimeur; mais ils l'ont colonisé. Un regard rétrospectif aurait tendance à concevoir le fascicule de façon carentielle, comme un livre trop petit, comme un maigre substitut, un livre de poche du pauvre. Là encore, quel que soit le bien-fondé de cette conception, elle entraîne malheureusement une cécité partielle, ici sur l'acte de lecture.

Le format fait tout d'abord partie des stratégies de facilitation de la lecture; pour un faible lecteur, il encourage préalablement la performance: oui, on peut en venir à bout, cette histoire n'a que 32 pages, ce n'est pas un gros bouquin intimidant par son épaisseur; d'ailleurs l'illustration l'éloigne encore de l'apparence du livre fétichisé par la grande culture. La désignation des fascicules par leurs lecteurs n'était pas du tout inspirée par une conception carentielle du livre; roman dont le faible lecteur peut intellectuellement prendre la mesure, et que le faible acheteur peut se payer, "roman à 10 cents": dévaluante pour la culture légitime, la désignation était hypocoristique pour le lecteur sériel. Pourtant, comme le gros bouquin, le fascicule est rempli d'action(s); entre dialogues et récit, pas le temps de décrire; au lecteur d'imaginer à partir de succintes indications locatives. Comme le livre, le fascicule constitue un tout; mais, contrairement au livre Belleslettres, protégé par son paratexte - c'est-à-dire par du texte mis sur la couverture pour intimider le faible lecteur -, l'appeau est ici une 
image. Et la couverture, aussi souple que le papier des pages, signale - mais faiblement - le début et la fin de l'objet-fascicule.

En fait, dans cette lecture, quel est l'équivalent du livre? quelle est donc l'unité pertinente? Le fascicule? la série? On aurait pu supposer que la volonté de fidéliser le lecteur aurait conduit spontanément auteurs et éditeurs vers la solution du récit à suivré, du classique feuilleton, dont chaque fascicule aurait constitué un épisode. N'était-ce pas justement la fonction de leur numérotation que d'annoncer la successivité? l'enchaînement?

$\mathrm{X}-14$ revint de sa commotion et reprit ses sens. Après avoir été bâillonné puis chloroformé sur la route il avait été jeté dans une cave sans air et sans lumière comme un paquet de guenilles. Combien de temps resta-t-il évanoui? il n'aurait su le dire [...]. (p. 1)

Ainsi débute Un nazi maudit ${ }^{29}$, neuvième épisode du "Génial espion X-14, agent secret canadien. Le lecteur est jeté dans une action évidemment en cours par ce topos in medias res, et, au cas où il n'aurait pas lu les épisodes précédents, le sommaire le met concisément au parfum. Il comprend l'essentiel: qu'il prend un train en marche, une aventure déjà en cours, et que les repères chronologiques ne sont pas déterminants, puique le héros luii-même peut continuer à prendre l'initiative malgré un flou dans son emploi du temps entre le fascicule de la semaine précédente et celui-ci. Après le mot "fin ", apparaissent les classiques questions du roman-feuilleton:

X-14 reviendra-t-il à la santé?

Sa figure sera-t-elle marquée?

Aura-t-il l'occasion de se mesurer de nouveau avec Fritz?

C'est ce que nous apprendrons au prochain épisode qui parâtra sous peu. Surveillez le dixième épisode. (p. 29.)

Toutefois, ces épisodes n'ont jamais fait l'objet d'une édition en volume comme c'était très souvent le cas des feuilletons de' la littérature populaire; à l'inverse, la formule même du roman sérialisé publié dans un magazine ou un journal (que sa première édition se soit faite sous forme de livre ou sous forme de roman-feuilleton), cette formule était complètement en déclin, alors qu'elle avait connu son heure de gloire plus tôt dans le siècle.

Ailleurs, le récit commence et s'achève par d'explicites et rudimentaires chevilles avec les fascicules encadrant ce numéro ${ }^{30}$. En fait, le

29. Anonyme, Un nazi maudit, Berthierville, Editions Irène, coll. -L'espion X-14 •, . $n^{\circ}$ 9, [s.d.].

30. Par exemple dans L'Heure zéro (coll. - Aventures fantastiques de l'agent ZED-29, l'as des as du contre-espionnage canadien *, $n^{\circ}$ 201) de M. Leroux: : $\hat{A}$ peine ZED- 
plus souvent, l'histoire de chaque fascicule est bouclée sur elle-même; dans Mission de mort, l'enquête menée par le héros pour découvrir le traître est encadrée en amont par le bombardement des troupes canadiennes en Corée par un avion canadien (adunaton situationnel) et en aval par le massacre des clandestins communistes par le seul Espion $\mathrm{n}^{\circ} 13$; et à ce bouclage narratif se superpose le bouclage rhétorique habituel de l'annonce d'une autre mission. Bouclée sur elle-même? Presque.

Pour le lecteur, ce qui commande les scansions lecturales peut correspondre à l'unité commerciale-fascicule, mais pas nécessairement. La totalité d'une série est à peine une entité pour l'éditeur (qui vit plutôt de semaine en semaine), pas toujours pour l'auteur (un héros pouvait continuer à vivre des aventures alors que plusieurs auteurs se succédaient sous un même pseudo, voire sous des signatures différentes), et encore moins pour le lecteur (surtout pour une série longuissime comme IXE-13). La portion de la série formant l'histoire suivie aura donc une définition différente selon que l'on se place du côté poiétique ou du côté esthésique, et même, du seul côté de l'émetteur, une longueur variable, depuis 1 jusqu'à $n$ fascicules, $n$ pouvant être la somme de tous les fascicules de la série.

Porosité relative du paratexte donc, le lecteur enchaînant sur le fascicule suivant (l'éditeur le pousse à ça); mais il n'enchaîne que de façon paresseuse, nonchalante. La dépendance du lecteur sériel à son intoxication n'est pas toujours aussi exigeante, aussi faut-il prévoir qu'il puisse revenir à la lecture après une périodè de négligence, d'éloignement, sans lui avoir fait manquer un événement crucial pour la compréhension de l'histoire globale.

Ce type de lecture est malheureusement mal compris dans les études sur la lecture, effectuées généralement par de forts lecteurs. Il n'est en effet pas simplement déficient par rapport à la lecture attentive, suivie, portant sur de longues unités textuelles (plusieurs chapitres, voire un roman entier lus d'un seul coup); il se recommande en effet par au moins un trait singulier: ce type de lecture n'a pas de modèle dans la littérature ni dans les pratiques scolaires d'apprentissage de la lecture ou de l'écriture.

Question insolite, dès lors: d'où auteurs et lecteurs avaient-ils tiré cette idée de l'œuvre à géométrie variable? Du côté des auteurs, une chose est avérée, l'influence des romans français ou britanniques en la

29 eut-il accompli sa mission qu'on le laņ̧a dans une autre. (p. 1); - Mais une autre miśsion l'attendait. Qu'était-ce donc?. (p. 31). 
matière était faible; celle de la pratique de l'espionnage ou du discours sur l'espionnage réel, nulle. Aussi, pourquoi ne pas aller chercher un modèle à l'œuvre à géométrie variable dans les médias de masse du temps? Cherchons un type d'œuvre de large diffusion, offrant à la fois une unité quasi bouclée sur elle-même et un principe intégrateur faisant tenir ensemble plusieurs de ces unités dans un propos narratif plus vaste, une ouvre assujettie aussi à la nécessité de mouliner de l'intensité émotionnelle...

Et nous trouvons un produit typiquement hollywoodien aujourd'hui disparu, mais depuis les années quarante distribué assez largement au Québec par le réseau de salles paroissiales: le serial! L'esthétique du serial se fondait sur une nécessité fondatrice et trois composantes obligées, trois principes de liaison diégétique: l'enchaînement d'actions rebondissant, continu, inlassable; le Wbodunit, code herméneutique formant l'ossature de chaque film du serial; le Cliffbanger, punch suspensif censé exciter l'envie de connaître la suite, de venir voir la semaine suivante la séquelle du film ainsi interrompu; le McGuffin, liant global, code herméneutique n'ayant souvent même aucune incidence en retour sur ce qui se sera passé entre l'ouverture de ce code et le moment où le spectateur découvre qui est derrière le masque de Zorro, derrière l'armure de Don del Oro ${ }^{31}$...

Si cette hypothèse de l'influence cognitive du serial sur l'écriture et la lecture de la paralittérature québécoise par fascicules est fondée, seraient éclairées sous un nouveau jour à la fois l'économie des descriptions (dans le serial, l'image y supplée) et la spontanéité avec laquelle le lecteur de fascicule se faisait son propre cinéma à partir des sollicitations du 32 pages.

\section{Études littéraires et récits fasciculaires}

Ainsi, que, pour le meilleur ou pour le pire, le Québec ne dispose pas de services secrets n'aura pas empêché toute une armée de l'ombre pure laine, portant des noms de code directement issus d'une bataille navale entre potaches, de s'agiter entre complots communistes, péril jaune, coups sournois, repos du guerrier... et entre les feuilles de papier news des fascicules d'avant-hier, pour la plus grande joie d'un lectorat aujourd'hui disparu: IXE-13, UZ-16, AC-12, agent $Z$, ZED-29, K-7, X-14, espion $n^{\circ} 13 \ldots$

31. Sur le serial $c f$. R. Lacourbe, "Un monde fou, fou, fou ou l'étrange univers du serial (1929-1956). 1" épisode ., Ecran, n 68, avril 1978. 
Mais que cette armée ait existé ne l'aura pas empêché non plus de disparaître de la mémoire sinon de ses lecteurs; du moins de celle de l'institution littéraire. Oubli? En fait, il aura fallu attendre les années quatre-vingt et le travail d'une équipe de l'Université Laval, autour de Denis Saint-Jacques, pour que l'espionnage de fiction québécois sorte des oubliettes de l'histoire littéraire ${ }^{32}$; et ces spécialistes diagnostiquaient moins un oubli de la part de l'institution qu'un choix plus délibéré de ne pas vouloir savoir. Artistes et intellectuels n'ont jamais eu grande sympathie pour la culture de masse, qui privilégie le commun dénominateur esthétique à l'originalité individuelle, qui se fonde sur la demande et l'industrialisation de la production culturelle plutôt que sur le génie et la communauté des gens de goût... Le face-à-face n'est ni nouveau ni particulier au Québec.

Le fait d'en parler depuis une revue littéraire risque de produire un effet pervers, en laissant croire qu'il pourrait s'agir de réhabiliter, de rendre acceptable cet espionnage fasciculaire. En fait, ce dernier se caractérise tout aussi bien par l'indifférence totale qu'il rendait à l'institution littéraire et à ses valeurs. Au-delà du choix de trouver plus déterminante l'existence de cette paralittérature que sa valeur ${ }^{33}$ - choix qui nous conduit à un travail préalable de répertoriage et de conservation $^{34}$ - les études paralittéraires doivent prendre en considération cette double et réciproque indifférence. Ainsi, tenter de comprendre l'espionnage fasciculaire, c'est peut-être d'abord trouver son niveau de pertinence historique adéquat. Puis, après avoir rappelé le rôle du feuilleton radiophonique dans l'émergence de ce phénomène paralittéraire, la place de l'illustration fasciculaire dans l'histoire de la figuration narrative, la promotion d'une théorie spontanée de la langue entiè-

32. Cf. surtout le volume collectif Le Pbénomène IXE-13, Québec, Presses de l'Université Lavál, coll. . Vie des lettres québécoises, 1984.

33. À la permanence et l'omniprésence du patron argumentatif littérature/paralittérature, faut-il aussi ajouter l'universalité, puisqu'il aura naguère suffi de rappeler l'existence d'une littérature de grande diffusion dans les années 1940-1960 pour qu'il soit activé de nouveau?

34. Deux premiers répertoires de la production fasciculaire, Petite Mémoire, petite bistoire "Petit-Format", Répentoire bibliographique de la collection .Petit-Format. des Editions modernes, et Jongleries, séries es collections, Répertoire bibliographique des Editions Paris-Tour-Eiffel de Montréal doivent prochainement paraitre; en outre, Richard Saint-Germain est l'auteur d'un relevé bibliographique préliminaire mais déjà indispensable, Le récit populaire au Québec de 1940 à 1960: premier répentoire des maisons d'édition, des collections, des séries et des titres publiés, 1989, et de quelques études particulières sur - La collection Petit-Format des Éditions de La Revrue Moderne de Montréal,, loc. cit., .Le Québec 110 cents. Une époque opaque ,, Cabiers pour la littérature populaire, n* 8-9, automne 1987, et .Une industrie québécoise: le "rejaquettage ", Voix \& Images, vol. XV, n 2, hiver 1990. 
rement communicationnelle correspondant à une pratique textuelle aux antipodes du fétichisme culturel, l'interpénétration des pratiques culturelles vues comme simples avatars du Récit paralittéraire, les transferts cognitifs dans les opérations de compréhension, c'est sans doute l'assigner à une histoire des médias de masse.

Déplacement de perspective qui servira de préalable à une interprétation globalisante du thème des services secrets québécois, de ses formes, de son institution et de son lectorat - par la figure de la déterritorialisation ${ }^{35}$.

35. Paul Bleton et Richard Saint-Germain, -Culture fasciculaire et déterritorialisation. Espions mais aussi aventuriers, détectives \& amoureuses du Québec paralittéraire des années 40-60:, à paraître. 